\section{Physics of Backscattered Electron Diffraction}

Patrick Camus, NORAN Instruments Inc.

\section{Background}

The scale of features that control the physical properties of engineered materials is ever decreasing. We can investigate the structure of these materials using several techniques. The techniques are usually selected based on the feature size of interest. Typical scales are the millimeter or optical scale, the micron or microscopic scale, and the nanometer or atomic scale. As the feature size decreases, the capabilities of the technique must increase.

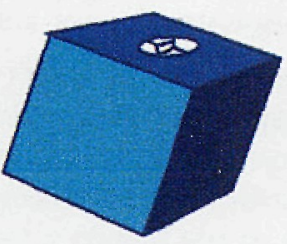

Millimeter Scale

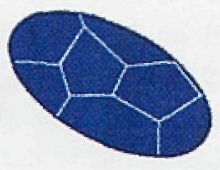

Micron

Scale

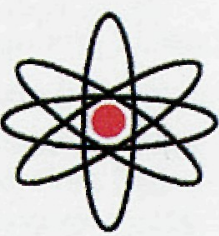

Nanometer Scale
Typical features that require at least microscopic characterization include particles in super alloys, grain misorientations in high temperature superconductors and thin films, inclusions in steels, reaction products in gas chambers.

Real-space imaging is the typical method used to investigate materials features, but spectroscopic and diffraction techniques are also quite useful for characterization. Electron backscattered diffraction (EBSD) is a phenomenon that was described in 1928 by S. Kikuchi, It occurs when high-energy electrons are elastically scattered by the atomic planes of a crystalline sample. The geometric source of the electrons is not the electron beam but the backscattering volume within the sample. The diffraction pattern results from the interaction of the backscattered electrons escaping from within a few hundred nanometers of the sample surface and the crystal planes through which they pass.

\section{Crystallography}

Most metals, semiconductors, and ceramics consist of periodic arrays of atomic clusters. These clusters, or cells, can be thought of as the fundamental building units of the material. Repeated stacking of the cells produces a sample whereby each cluster origin within the sample has the same atomic environment. From a geometric viewpoint, an infinite number of planar arrangements can be determined within this crystalline volume. The spacing of these planes can be related to the spacing of the atoms in the clusters and the cell size. Identification of the elemental species and the location of the atomic positions within the clusters and cells uniquely identify the phase type of the sample.

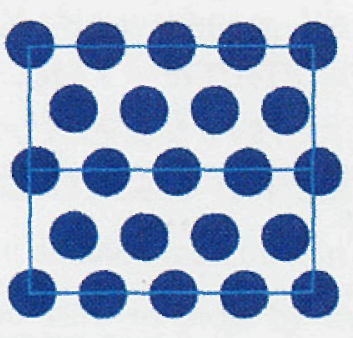

Atomic Stacking

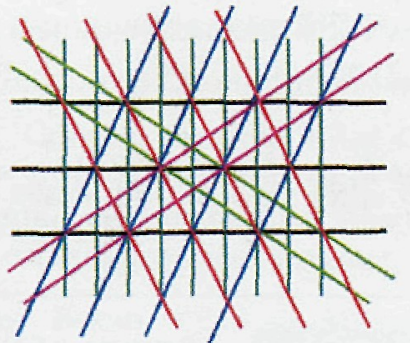

Atomic Planes

\section{Diffraction}

Diffraction is a technique whereby waves interact with a grating and constructively interfere. This interference is manifest in the measurement and acquisition of a diffraction pattern. Any periodicities present in the diffraction grating are represented in the diffraction pattern if the wavelength of the wave is of the same order as the grating spacing. To investigate the atomic arrangements in crystals, wavelengths on the scale of less than a nanometer are required. Fortunately, $x$-rays and high energy electrons have wavelengths of this scale and can be used to analyze crystals.

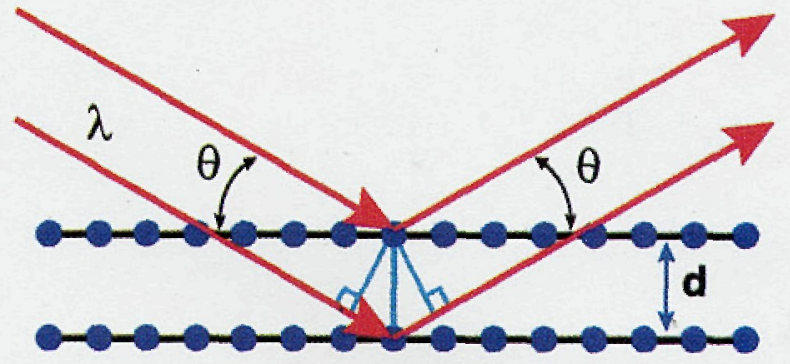

Constructive interference of waves, i.e., the diffracting condition, is defined by Bragg's Law:

$$
n \lambda=2 d \operatorname{Sin}(\theta)
$$

where $\lambda$ is the wavelength of the waves, $d$ is the spacing of the grating, and $\theta$ is the Bragg angle for that spacing. Whenever the Bragg condition is obtained, a high intensity is obtained at the detector. At small angular deviations from the diffracting condition ( $<1$ degree), the intensity drops to nearly zero. Thus, a pattern is obtained with dimensions directly related to the grating. In the case of crystals, the grating is the atomic planes that are uniquely described by the diffraction pattern.

If the wave source is a small-diameter beam impinging on a single crystal, as for TEM and XRD, a spot pattern is observed. If the source is a point or volume with nearly uniform intensity, the pattern consists of a series of lines. Lines, instead of points, arise due to the wide range of lateral incidence angles of the waves diffracting from the planes.

\section{Kikuchi Pattern Formation}

When backscattered electrons pass through a crystal, they interact with its atomic planes. Because of the point nature of the electron source, the electrons may interact with either the "top" or the "bottom" of the planes. There are then two diffracting conditions for each plane leading to two lines in the pattern for each plane.

Specimen Tilt

The priority of pattern measurement is to have as great a signal-to-noise level as possible. This typically occurs when the signal is optimized. For backscattered electrons, the maximum intensity occurs when the sample is tilted at angles greater than 50 degrees from horizontal. Thus, high samples tilts, while not required for $E B S D$, are required for the highest quality images.

\section{Geometry}

The imaging phosphor screen is positioned in the chamber to collect images of this high-intensity region. The typical arrangement is to mount the screen axis perpendicular to the incident beam direction. The specimen-to-screen distance, comparable to the diffraction camera length, determines the magnification of the pattern. The pattern center is defined as that position on the screen whose normal points to the beam incidence location on the specimen. Accurate analysis of a pattern requires that the geometry be calibrated to known distances. This requires that the screen and sample positions be fixed in space from one analysis to another.

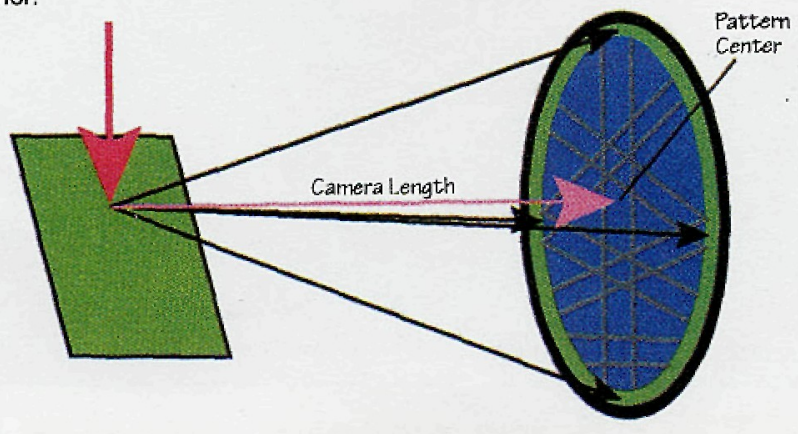


Indexing software algorithms have been developed to automatically analyze the resultant patterns and determine the sample orientation. If the crystal structure of the feature of interest is known a priori, then a 2D map of these orientations may be performed. This results in a texture map of the crystals. If the structure of the feature is completely unknown but the elements present are identified, an automated database search will narrow the possible compounds to a limited few for final determination. In this way, the phase identification of the feature may be determined.

\section{Image Collection and Processing}

The angular intensity distribution of backscattered electrons from a specimen is not uniform but follows an approximately cosine distribution. Since the Kikuchi lines are subtle intensity features on a background field, high-quality images require flat field intensity correction. This procedure removes the non-uniform background intensity which increases the signal-to-noise ratio (contrast). This processing ultimately permits easier feature recognition both visually and via software.

\section{Summary}

EBSD is a very useful technique for determining the orientation and crystal structure of crystalline solids on the sub-micron scale. It is easily performed in an SEM and therefore can provide many laboratories with phase identification and characterization capabilities without resorting to more expensive microscopic and microanalytical techniques.

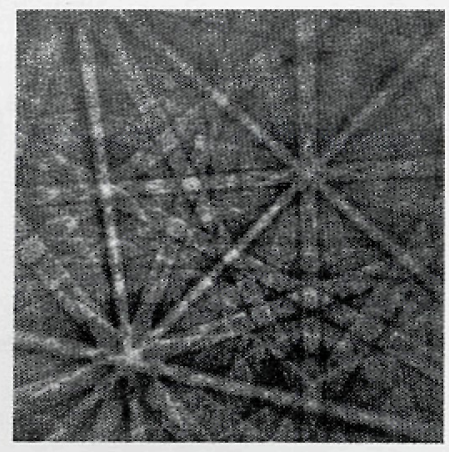

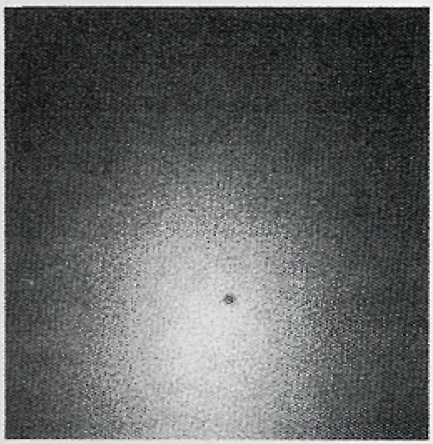

Background Image

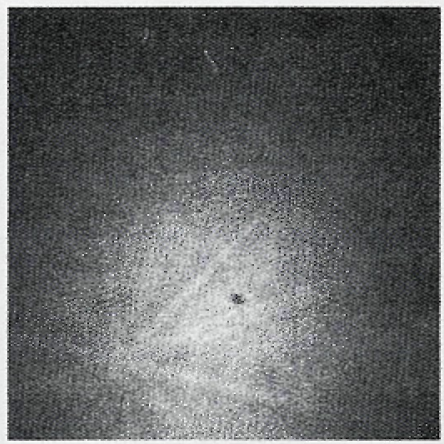

Original Pattern

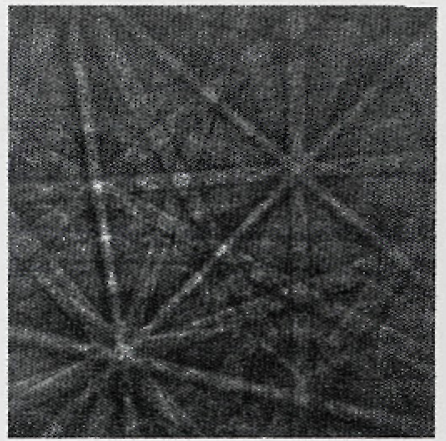

Flat-Field Corrected Pattern

\section{Introducing $\cdots \quad$ S E M I C A P S}

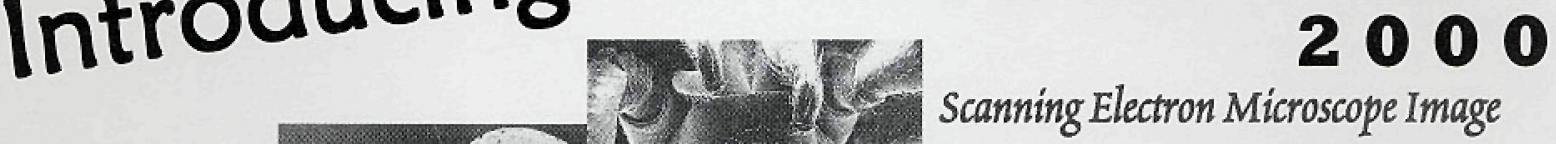

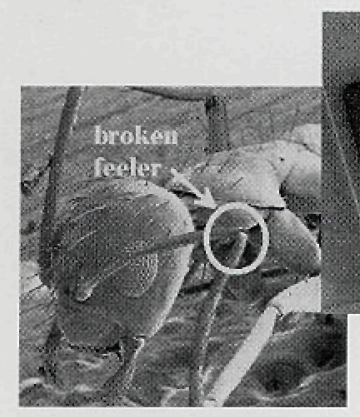

A new release in the A Windows $^{\text {Tx }}$ environment, SEMICAPS 2000 digitizes images from SEMs, TEMs, Augers, FIBs and light microscopes into PC compatible file formats. Its simple menu driven user interface makes learning easy and no prior experience is required.

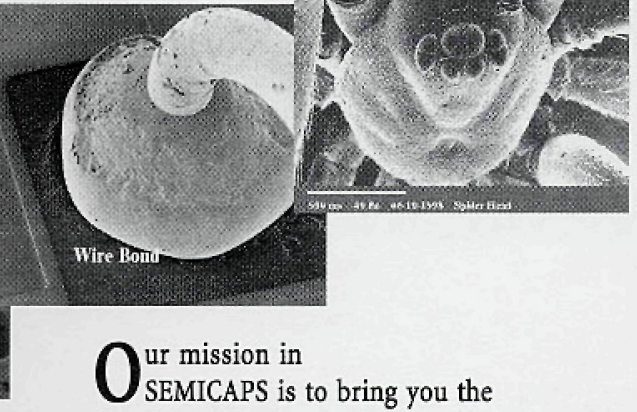

latest in this arena and to provide you with the highest satisfaction, covered with our money-back guarantee. With our commitment and expertise, SEMICAPS 2000 is undoubtedly your very wise choice for cutting edge imaging technology.

\section{Collection and Processing System}

$\nabla$ Image Archival

$\nabla$ Hardware Noise Reduction

$\nabla$ Advance Image Processing

$\nabla$ Feature Measurement

Annotation

Customized Report Printout

\section{Call (408) 986-0121}

\section{SEMICAPS Inc}

$2116 \mathrm{~B}$ Walsh Avenue,

Santa Clara CA 95050, USA

Phone : (408) 986-0121 Fax : (408) 986-1059

S E M I C A P S Email semicapseal.com

http://www.semicaps.com,sg 


\section{McCrone Research Institute}
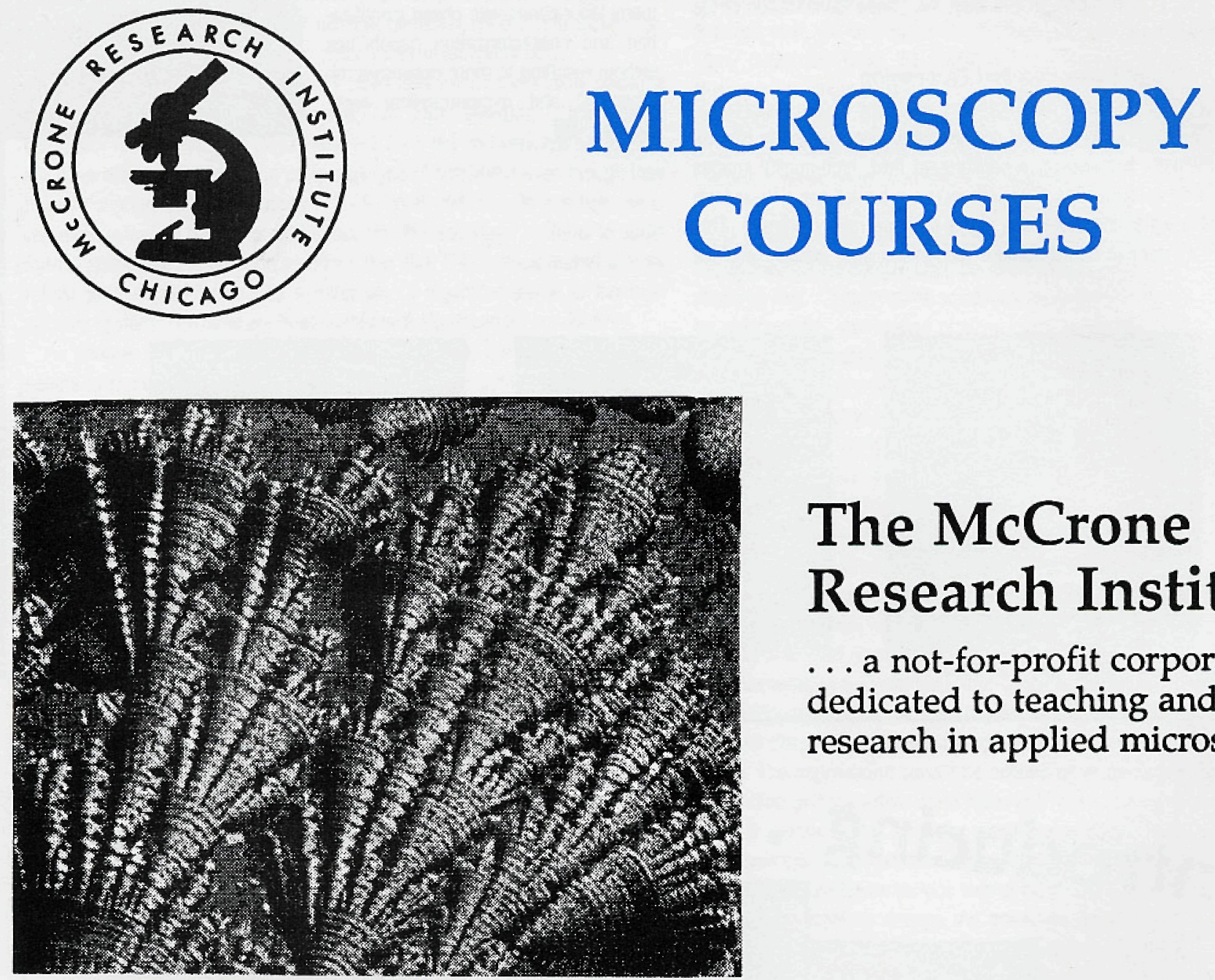

The McCrone Research Institute

... a not-for-profit corporation dedicated to teaching and research in applied microscopy

The McCrone Research Institute

- TEACHES MORE THAN 70 INTENSIVE COURSES EACH YEAR

- HAS SIX FULL-TIME TEACHING FACULTY

- PUblishes The Microscope (A QUARTERLY JOURNAL)

- HOSTS INTER/MICRO, AN ANNUAL INTERNATIONALLY-RECOGNIZED MICROSCOPY MEETING 


\section{Chicago Campus}

The Institute is fully equipped with more than 100 polarized, phase and other light microscopes, as well as SEM and FTIR microscopes, hot and cold stages, a 3000 volume library, reagents and all of the accessories necessary for critical applied microscopy.

\section{Applied Microscopy}

Our intensive courses, usually one week in length, are designed to provide practicing scientists with training in critical applied microscopy. Some courses provide an overview and emphasize the proper use of the microscope and its accessories. Other more specific courses are offered for chemists, biologists, criminalists, mineralogists, polymer chemists, art conservators, environmental pollution experts, contamination control analysts and quality control specialists.

\section{Hands-On Teaching}

Each course has lectures, demonstrations and hands-on laboratory practice so that students learn each technique by hearing about it, watching it being done and then doing it. Live video is used extensively for lectures and classroom demonstration. Students learn powerful and effective methods for studying, characterizing and identifying materials of all kinds, and for rapidly solving research, production, and quality control problems.

See our web site for the Chicago course schedule... or call to set up a CUSTOM-DESIGNED COURSE

at your own laboratory.

\section{APPLIED}

MICROSCOPY COURSES

POLARIZED LIGHT

PHASE CONTRAST

FTIR, SEM, TEM

FOR THE FIELDS OF:

MATERIALS SCIENCE ASBESTOS

BOTANICAL TRACES

ENVIRONMENTAL

FORENSIC SCIENCE

CONTAMINATION

PHARMACEUTICS

POLYMER SCIENCE

$\mathrm{QA} / \mathrm{QC}$

ART CONSERVATION

\section{FOR DETAILED INFORMATION CONTACT:}

The Registrar, McCrone Research Institute, 2820 South Michigan Avenue, Chicago, IL 60616-3292 PHONE: 312/842-7100 FAX: $312 / 842-1078$ E-MAIL: ndaerr@mcri.org 